\title{
La perspectiva ecológica en la investigación comparada
}

\author{
Mascarell Pérez, Ernesto \\ Escuela Municipal de Balonmano, Tegueste. España \\ Mascarell1960@hotmail.com
}

\section{Resumen}

En las últimas décadas la ecología y la teoría de los sistemas complejos se han configurado como un nuevo paradigma científico en el campo de la biología y las ciencias sociales (Sandin, 2006; Goodwin, 2007; Hilbert, 2016). Sin embargo, estos avances en el conocimiento, no se han trasferido al ámbito educativo. La investigación educativa continúa apoyándose en análisis unifactoriales o multifactoriales simples, mecanicistas y universales que describen la realidad y explican los hechos como una correlación estadística lineal de causa efecto (Sternberg, 1997; Feyerabend, 2002). La educación comparada no ha permanecido al margen de esta moda reduccionista y determinista, en parte por la popularidad de las certezas absolutas y en parte por las grandes oportunidades económicas y de promoción profesional que para los docentes ofrecen las soluciones rápidas (Apple, 2002; Stobart, 2006). Sin embargo, la realidad es que ni los problemas educativos son simples ni las soluciones pueden ser instantáneas (Esteve, 2001; Sidorkin, 2016). Por ello, consideramos que la investigación educativa necesita dejar a un lado las explicaciones deterministas y universales incorporando y aplicando nuevas metodologías, que facilite la comprensión de los procesos educativos en el contexto global y a la vez singular en el que se desarrollan.

\section{Abstract}

In recent decades, ecology and the theory of complex systems have been configured as a new scientific paradigm in the field of biology and social sciences (Sandin, 2006; Goodwin, 2007; Hilbert, 2016). However, these advances in knowledge have not been transferred to the educational field. Educational research continues to rely on unifactorial or multifactorial simple, mechanistic and universal analyzes that describe reality and explain the facts as a linear statistical correlation of cause and effect (Sternberg, 1997; Feyerabend, 2002), Comparative education has not remained outside this fashion reductionist and deterministic, partly because of the popularity of absolute certainties and partly because of the great economic opportunities and professional promotion that offer fast solutions for teachers (Apple, 2002; Stobart. 2006). However, the reality is that neither educational problems are simple nor solutions can be instantaneous (Esteve, 2001; Sidorkin, 2016). For this reason, we believe that educational research needs to put aside deterministic and universal explanations incorporating and applying new methodologies, which facilitate the understanding of educational processes in the global context and at the same time, in which they develop.

Palabras clave: ecología, complejidad, investigación comparada, mejora educativa.

Keywords: ecology, complexity, comparative research, educational improvement.

\section{INTRODUCCIÓN}

Una de las paradojas más grandes en el campo de la investigación científica en general y de la investigación en educación comparada en particular es que con frecuencia nos olvidamos de las preguntas fundamentales sobre ¿Por qué y Para qué investigamos? La tendencia a dar por supuesta u omitir la pregunta nos conduce al callejón sin salida de investigar por investigar en un círculo irracional de insatisfacción de resultados, que terminan por naturalizar la exclusión educativa y social. El problema de las preguntas se extiende a las metodologías habida cuenta de que estas se diseñan en función de la finalidad adoptando básicamente dos enfoques uno empírico y otro teórico (Adamson, Bray y Manson, 2010). 
En el enfoque empírico la investigación toma como referencia una característica o estado concreto del sistema y se compara con la de otro o del mismo en otro periodo de tiempo, haciendo una inferencia del resultado de la comparación, que será más precisa y objetiva cuanto más aislado y limitado sea el estudio.. Este procedimiento que resulta muy eficiente y rentable suele ser inapropiado en el ámbito de las ciencias sociales. En primer lugar, porque, los tiempos y espacios en un sistema sociocultural cambian continuamente haciendo que las medidas puntuales y los criterios universales sean ineficaces a la hora de explicar y predecir su comportamiento. En segundo lugar, porque no se puede establecer un criterio de valoración objetivo sin considerar y ponderar el peso de la singularidad en ese comportamiento o lo que es lo mismo el de las expectativas e intenciones de los distintos agentes, siendo habitual, que en las investigaciones educativas, se omitan las experiencias, relaciones y motivaciones de estos.

Por el contrario, el enfoque teórico parte de una descripción fundamentada del sistema objeto de estudio, en base al cual se decide con posterioridad, la metodología de investigación, los instrumentos y las técnicas necesarias para llevarla a cabo. En este caso nos encontramos con un problema distinto el de la dificultad de elaborar una definición estable y universal que describa la naturaleza compleja y dinámica de un sistema biológico y cultural, lo que nos conduce irremediablemente al terreno pantanoso de la intencionalidad y relatividad.

En ambos casos y con independencia del enfoque que se adopte muchos de los principios teóricos y metodológicos no se cuestionan, aceptando como verdades universales algunas proposiciones falsas, o cuanto menos muy discutibles. Para facilitar la comprensión de este problema, abordaremos dos variables recurrentes en la evaluación de los sistemas educativos, que se consideran objetivas, pese a carecer de razones fundamentadas para ello: la inteligencia de las personas y la excelencia de las instituciones. (Sternberg, 1997).

En primer lugar tenemos el problema de la validez, para que un instrumento o técnica de medida se considere valido es necesario que se demuestre la relación entre el mismo y lo que se supone que mide. Hasta la fecha nadie ha podido establecer la relación entre estos y la inteligencia y excelencia por el simple hecho de que nadie ha sido capaz de definir universalmente lo que son. Y la explicación es bien sencilla existen tantas inteligencias y excelencias como demandas y contextos de aplicación.

En segundo lugar tenemos el problema de la fiabilidad; la inteligencia y la excelencia hacen referencia a una manifestación y como tal, necesita expresarse o materializarse para ser valorada. El problema es que la expresión de la misma en la realidad nunca es igual, debido a que las situaciones y los problemas cambian a medida que los afrontamos; y con ello también la pertinencia y el valor de las respuestas y soluciones a los mismos. Por lo tanto, para que un método, instrumento o técnica sean fiables deberían incorporar alguna fórmula de ajuste que permitiera contemplar y ponderar estos cambios. Unas condiciones que por el momento no han logrado cumplir y que explicaría por ejemplo la inconsistencia de los resultados e incongruencia de las explicaciones que arrojan muchos de los test de inteligencia e informes de evaluación educativa. (Flynn, 2009; Monarca, 2015; Hampshire 2016).

\section{LA COMPLEJIDAD EN LA INVESTIGACIÓN COMPARADA}

Lo que hoy sabemos respecto a la educación es que es un sistema sociocultural complejo y que como tal debería estudiarse conforme a las propiedades que los caracterizan (Hilbert, 2016):

Orientación: Los seres vivos y sus sociedades son sistemas intencionales y por tanto tienen una orientación finalista, con independencia de que esta sea consciente o inconsciente. Por lo que en toda investigación deberían hacerse explícitos contemplando tanto los intereses del investigador como los del objeto de estudio.

Conexión: los sistemas están compuestos por elementos interdependientes de tal forma que no tiene sentido intentar explicar el desarrollo de alguno de ellos sin contemplar sus relaciones con los demás.

Jerarquización: los componentes de los sistemas complejos se organizan en niveles especializados atendiendo las necesidades y demandas en un determinado ámbito. Algo a tener presente a la hora de establecer y ponderar las variables más pertinentes, determinar las causas y atribuir responsabilidades. 
Auto-organización: a diferencia de los sistemas mecánicos en los sistemas orgánicos sus componentes operan simultáneamente sin una dirección centralizada, siguiendo un patrón no lineal que implica tanto fases de ajustes y equilibrios evolutivos como de desajustes y desequilibrios involutivos, siendo esta alternancia de fases su proceso natural de desarrollo De ahí que en la investigación y evaluación de los sistemas debamos prestar especial atención a su historia para descubrir los patrones y la Zona de desarrollo próximo (Vygotsky, 1979).

Singularidad: no existen ni pueden existir dos sistemas o seres iguales, porque la experiencia vital implica un cambio continuo, de ahí la diversidad de formas y relaciones que se aprecian en la naturaleza. Por lo tanto en las investigaciones se debería contemplar esta diversidad a la hora de universalizar y trasferir los resultados.

Adaptabilidad: la actividad vital de un organismo se corresponde con un proceso interactivo entre los intereses capacidades y expectativas internas del organismo y las externas del medio, sin conocer las características de estas interacciones resulta difícil predecir su comportamiento y descubrir los puntos o fases críticas en las que se puede estabilizar cambiando sus propiedades y orientación.

Equifinalismo y estocástica: el desarrollo de un sistema dependen del camino que recorre, de tal forma que por un lado, pequeñas experiencias pueden dar lugar a grandes cambios con resultados impredecibles y por otro que un mismo resultado o conclusión se pueden obtener por caminos diferentes La realidad por tanto es fruto de una cadena histórica de interacciones singulares, que no se puede explicar estudiando simplemente una variable aislada en un momento puntual.

Emergencia. Los sistemas orgánicos se desarrollan y evolucionan en su complejidad creando nuevas formas y entidades con características que no solo no tenían sus componentes originales sino que se orienta conforme a unos intereses y fines diferentes. Estudiar los mismos, por tanto, es estudiar las implicaciones entre esta diversidad de intereses a diferentes niveles.

Contemplar la educación como un sistema complejo tiene enormes implicaciones en el ámbito de la investigación comparada, ya que obliga a un cambio de paradigma, y con ello a la necesidad de revisar en profundidad los fundamentos teóricos y prácticos de las ciencias de la educación. Los resultados de esta revisión que viene desarrollándose desde hace décadas comienzan a materializarse poniendo en cuestión algunos mitos hegemónicos:

En cuanto a la psicología del desarrollo, hecha por tierra las ideas de Piaget y Kohlberg que concebían el desarrollo humano como un proceso predeterminado, acumulativo y lineal de maduración de capacidades físicas, intelectuales y morales individuales; una vez demostrado que el crecimiento y la maduración son procesos complejos, multidimensionales, interdependientes y no lineales, de tal forma que comprende tanto periodos involutivos como evolutivos, que además se alternan continuamente a lo largo de toda la vida. (Smith \& Thelen, 1993) Por ejemplo existen diferencias de hasta cuatro años en la maduración fisiológica entre adolescentes con una misma edad cronológica. Unas diferencias sustanciales que no solo inciden en las capacidades físicas sino también en los intereses, relaciones y desarrollo intelectual de los alumnos, y que sin embargo se pasan por alto y no se contemplan en la investigación escolar (Blakemore, Burnett, \& Dahl, 2010; Gómez, 2013)

En lo que se refiere a la teoría de la educación, ponen en tela de juicio tanto las tesis mecanicistas del conductismo como las reduccionistas del cognitivismo. El aprendizaje no es solo el producto de un proceso basado en estímulos y respuestas universales (Wherteimer, 1991; Tomasello, 2013) como tampoco se limita al procesamiento lógico de la información en el cerebro (Ornstein, 1993; Noe, 2010); es algo consustancial a la experiencia del humana por lo cual el problema del papel de la herencia y el ambiente en el aprendizaje está mal enfocado, ya que ambos son inseparables. La experiencia vital implica una modificación interactiva constante del ser y el entorno, por lo tanto la investigación sobre la herencia y la experiencia solo cobra sentido en la intención y la aplicación de la misma, algo que tendemos a pasar por alto en el campo educativo donde seguimos aplicando el sinsentido de una investigación pura intentando descubrir que fue primero la experiencia o la herencia. En realidad lo que esconden estas posturas en el mejor de los casos es un desconocimiento profundo sobre las ciencias de la educación y en el peor un malsano interés económico y profesional por vender remedios y soluciones teóricas y prácticas tan exclusivas como ineficaces.

Al hilo de lo expuesto y en lo concerniente a la evaluación educativa de la inteligencia y la excelencia debemos, por un lado considerar la diversidad de tiempos de aprendizaje y por lo tanto eliminar o al menos relativizar los resultados de las evaluaciones puntuales y selectivas (Esteve, 
2001; Sternberg, 2002); y por otro desechar de una vez por todas psicometría como instrumento de distinción y clasificación educativa una vez desacreditados e invalidados sus presupuestos sobre $\mathrm{Cl}$ y la anormalidad humana (Kincheloe, Steinberg, \& Villaverde, 2004; Hampshire, 2016) una tarea ardua habida cuenta de colosal negocio de las patologías educativas y la necesidad de legitimar políticamente la exclusión social (Bourdieu, 1988).

Por último en lo que se refiere a la Filosofía de la educación y más concretamente a sus interpretaciones: éticas estéticas y económicas, la practica educativa se empeña en caminar en el sentido opuesto a la ciencia, aferrándose a una visión de un ser humano egoísta por naturaleza que se desarrolla mediante la competencia inter e intraespecífica (Piaget, 2001; Kohlberg, 1992; Darwkins, 2000), de tal forma que los sistemas educativos actuales se orientan al desarrollo de un individualismo patológico y unas relaciones instrumentales e insolidarias que nos abocan a la infelicidad personal, la insolidaridad social y la irresponsabilidad ambiental. Y para hacerlo desatienden los últimos 40 años de investigaciones que corroboran nuestra naturaleza social y empática (laconomi, 2009; De Waal, 2013), nuestra preferencia por la búsqueda del bienestar común (Onstron, 2000; Zamagni, 2009) y el hecho básico de que el aprendizaje y el desarrollo humano es más producto de la cooperación y la simbiosis mutualista que de la competencia salvaje o el parasitismo (Tomasello, 2010; Bowles, \& Gintis, 2011).

\section{EL ANÁLISIS INTEGRAL EN LA INVESTIGACIÓN COMPARADA}

El hecho de que educación marche en dirección contraria a la práctica y la investigación educativa se debe principalmente a una falta soluciones alternativas a los problemas a los que se enfrenta, necesitamos actuar y si no se nos facilitan herramientas mejores seguiremos usando las conocidas y disponibles. En ese sentido propongo un modelo de análisis integral que permite la comprensión de un sistema social complejo a partir de la definición correcta de sus agentes / componentes a diferentes niveles y de los variables universales con las que operan. A continuación exponemos, a modo de ejemplo y de manera esquemática un modelo ecológico de descripción del sistema educativo (tabla 1).

\begin{tabular}{|c|c|c|c|c|}
\hline \multicolumn{5}{|c|}{ CONFIGURACIÓN DEL SISTEMA EDUCATIVO } \\
\hline Agentes & Tiempo & Espacio & Normas & Propósito \\
\hline $\begin{array}{c}\text { Administración } \\
\text { (político) }\end{array}$ & Ciclo vital & Sociedad & $\begin{array}{c}\text { Legislación } \\
\text { educativa }\end{array}$ & $\begin{array}{c}\text { Incluir } \\
\text { Rentabilizar }\end{array}$ \\
\hline $\begin{array}{c}\text { Centro Escolar } \\
\text { (director/gestor) }\end{array}$ & $\begin{array}{c}\text { Ciclos } \\
\text { educativos }\end{array}$ & $\begin{array}{c}\text { Comunidad } \\
\text { escolar }\end{array}$ & $\begin{array}{c}\text { Normas del } \\
\text { centro }\end{array}$ & $\begin{array}{c}\text { Integrar } \\
\text { Promocionar }\end{array}$ \\
\hline $\begin{array}{c}\text { Grupo de clase } \\
\text { (docente) }\end{array}$ & Curso escolar & Grupo de clase & $\begin{array}{c}\text { Normas del } \\
\text { aula }\end{array}$ & $\begin{array}{c}\text { Enseñar } \\
\text { Aprobar }\end{array}$ \\
\hline Alumno & Actividad & Individual & $\begin{array}{c}\text { Moral y } \\
\text { Valores }\end{array}$ & $\begin{array}{c}\text { Aprender } \\
\text { Mejorar }\end{array}$ \\
\hline
\end{tabular}

Una vez definida la estructura del sistema podemos analizar la dinámica de relaciones entre componentes tomando como referentes los roles e intenciones de sus agentes a distintos niveles (McGinn, y Welsh, 2001). Para ello podemos por ejemplo diseñar una tabla multinivel de doble entrada en la que relacionamos cada agente consigo mismo y con el resto. Este modelo de investigación nos permite, estudiar la interdependencia de los componentes de un sistema, descubrir los puntos de apalancamiento e incluso diseñar modelos teóricos de intervención en los que se pueden simular y comprobar los efectos que tendrían las decisiones y comportamientos de cada agente sobre la evolucion del sistema en su totalidad ${ }^{1}$ (Tabla 2).

\footnotetext{
${ }^{1}$ Utilizando un software de ARS podemos realizar el análisis y una representación gráfica de la red de relaciones visualizando los puntos críticos que limitan o afectan el desarrollo del sistema (Quiroga, 2003).
} 


\begin{tabular}{|c|c|c|c|c|}
\hline \multicolumn{5}{|c|}{ INTENCIONES DE LOS AGENTES PORTADORES DE INTERESES } \\
\hline Agentes & $\begin{array}{l}\text { Administración } \\
\text { Político }\end{array}$ & $\begin{array}{c}\text { Centro Escolar } \\
\text { Director }\end{array}$ & $\begin{array}{c}\text { Grupo de Clase } \\
\text { Profesor }\end{array}$ & Alumno \\
\hline $\begin{array}{l}\text { Administración } \\
\text { Político }\end{array}$ & $\begin{array}{c}\text { Mejorar los resultados } \\
\text { o cambiar el currículo } \\
\text { y la organizacion } \\
\text { escolar }\end{array}$ & $\begin{array}{c}\text { Mejorar la rentabilidad } \\
\text { o cambiar los centros } \\
\text { educativos }\end{array}$ & $\begin{array}{l}\text { Mejorar o cambiar la } \\
\text { formación selección } \\
\text { y designación del } \\
\text { profesorado }\end{array}$ & $\begin{array}{l}\text { Mejorar o cambiar } \\
\text { los criterios de } \\
\text { promoción y } \\
\text { selección del } \\
\text { alumnado }\end{array}$ \\
\hline $\begin{array}{c}\text { Centro Escolar } \\
\text { Director }\end{array}$ & $\begin{array}{c}\text { Mejorar la demanda } \\
\text { o cambiar la oferta } \\
\text { educativa del centro }\end{array}$ & $\begin{array}{c}\text { Mejorar los resultados } \\
\text { o cambiar la } \\
\text { organización del } \\
\text { centro }\end{array}$ & $\begin{array}{l}\text { Mejorar o cambiar } \\
\text { la distribución y } \\
\text { asignación del } \\
\text { profesorado }\end{array}$ & $\begin{array}{l}\text { Mejorar o cambiar } \\
\text { la distribución grupal } \\
\text { y asignación del } \\
\text { alumnado }\end{array}$ \\
\hline $\begin{array}{c}\text { Grupo de Clase } \\
\text { Profesor }\end{array}$ & $\begin{array}{c}\text { Mejorar los resultados } \\
\text { o cambiar de } \\
\text { asignatura y/o centro } \\
\text { educativo }\end{array}$ & $\begin{array}{c}\text { Mejorar las relaciones } \\
\text { o cambiar de grupo }\end{array}$ & $\begin{array}{c}\text { Mejorar las tareas o } \\
\text { cambiar las actividades }\end{array}$ & $\begin{array}{c}\text { Mejorar la } \\
\text { enseñanza o cambiar } \\
\text { la metodología }\end{array}$ \\
\hline Alumno & $\begin{array}{c}\text { Mejorar las } \\
\text { expectativas o } \\
\text { cambiar de estudios } \\
\text { y/o centro educativo }\end{array}$ & $\begin{array}{l}\text { Mejorar los resultados } \\
\text { o cambiar de } \\
\text { programa de estudios }\end{array}$ & $\begin{array}{l}\text { Mejorar las relaciones o } \\
\text { cambiar de grupo }\end{array}$ & $\begin{array}{c}\text { Mejorar el } \\
\text { aprendizaje } \\
\text { o cambiar la } \\
\text { estrategias }\end{array}$ \\
\hline
\end{tabular}

Esta metodología nos permite comparar los resultados de dos sistemas o del mismo sistema en dos periodos de tiempo distintos en base a su coherencia interna o lo que es lo mismo su capacidad y tendencia evolutiva o involutiva. De tal forma que se superan tanto el problema de la relatividad en las interpretaciones como los de reduccionismo temporal y descontextualización espacial que hasta hace poco tiempo planteaba el estudio de la complejidad.

\section{DISCUSIÓN Y CONCLUSIONES}

La investigación en educación en general y en educación comparada en particular no ha permanecido ajena, al abuso en la adopción al criterio hegemónico y predeterminado de la ciencia positivista dando como resultado la interpretación etnocéntrica y superficial de los problemas y la adopción de medidas o practicas universales que no permiten mejorar ni el conocimiento ni el funcionamiento de los sistemas. Pensamos que ha llegado el momento de salir de la zona de confort en la que se haya la investigación y asumir la complejidad de la realidad diseñando e incorporando nuevos métodos ecológicos de investigación que nos permitan comprender la compleja red de relaciones entre los agentes y el medio escolar y como esta puede modelarse para facilitar un desarrollo educativo basado en la co-evolución.

\section{REFERENCIAS BIBLIOGRÁFICAS}

Apple, M. (2002). Educar como Dios manda. Mercados, niveles, religión y desigualdad. Barcelona. Paidos Ibérica.

Blakemore, S. J. Burnett, S. Dahl. R. The role of puberty in the developing adolescent brain Human brain mapping 2010; 31 (6), 926-933.

Bourdieu, P. (1988). La distinción Criterio y bases sociales del gusto, Madrid. Editorial Taurus. 
Bowles, S., \& Gintis, H. (2011). A cooperative species: Human reciprocity and its evolution. New Jersey: Princenton University Press.

Bray, M.; Adamson, B. y Mason, M. (compiladores) (2010). Educación comparada. Enfoques y métodos. Buenos Aires, Granica.

Dawkins, R. (2000). El gen egoista Barcelona. Salvat Editores.

De Waal, F. (2013). La edad de la empatía. Barcelona: Tusquets Editores.

Esteve, J. M. (2001). Un examen a la cultura escolar. Barcelona. Octaedro.

Feyerabend, P. (2002). Contra el método. Esquema de una teoría anarquista del conocimiento. Barcelona: Ediciones Folio Flynn, J. (2009). ¿Qué es la inteligencia? Más allá del efecto Flynn. Madrid: TEA.

Gómez-Campos $\mathrm{R}$ et al. Valoración de la maduración biológica: usos y aplicaciones en el ámbito escolar. Rev Andal Med Deporte. 2013; 6(4):151-160.

Goodwin, B. (2007). Nature's Due: Healing Our Fragmented Culture. Edimburgh: Floris.

Gould, S. (2002). La falsa medida del hombre. Barcelona: Bosch

Hampshire, Roger R. Highfield, Beth L. Parkin, Adrian M. Owen. Fractionating Human Intelligence. Neuron, $2012 ; 76$ (6): 1225 DOI: 10.1016/j.neuron.2012.06.022

Hilbert, M. (2013). Charla sobre sistemas complejos sociales (CCSSCS). CEPAL. Chile http://www.martinhilbert.net/ccsscs-html/.

lacoboni, M. (2009). Las neuronas espejo. Empatía, autismo, imitación o de como entendemos a los otros. Buenos Aires: Katz Editores.

Kohlberg, L. (1992). Psicología del desarrollo moral. Bilbao. Desclée de Brouwer.

Kincheloe, J., Steinberg, S., \& Villaverde, L. (Comps.). (2004). Repensar la inteligencia: Hacer frente a los supuestos psicológicos sobre enseñanza y aprendizaje. Madrid: Editorial Morata.

McGinn, N. y Welsh, T. (2001). Hacia una metodología para el análisis de portadores de intereses. en Revista Latinoamericana de Estudios Educativos. vol. XXXI, núm. 1, $1^{\circ}$ trimestre. http://redalyc.uaemex.mx/redalyc/pdf/270/27031102.pdf

Monarca, H. (Coord.). (2015). Evaluaciones externas. Mecanismos para la configuración de representaciones y prácticas en educación. Buenos Aires: Miño y Dávila,

Noë, A. (2010). Fuera de la cabeza. Por qué no somos el cerebro y otras lecciones de la biología de la consciencia, Barcelona, Kairós.

Ornstein, R. (1993). Psicología de la conciencia: una exploración del comportamiento humano. Madrid. Edaf.

Ostrom, E. (2000). El gobierno de los comunes. La evolución de las instituciones de acción colectiva. Ciudad de México: Fondo de Cultura Económica.

Piaget, J. (2001). Psicología y pedagogía. Barcelona: Crítica.

Quiroga, A. (2003). Taller de ARS con Ucinet 6 y NetDraw avanzado Universidad Pompeu Fabra http://revista-redes.rediris. es/webredes/talleres/opbauci.pdf

Sandin, M. (2006). Pensando la evolucion, pensando la vida. Murcia. CAUAC / Crimentales.

Sidorkin, A. (2007). Las relaciones educativas. Educación impura, escuelas desescolarizadas y dialogo con el mal. BarceIona. Octaedro.

Smith, L. Thelen, E. (1993). A Dynamic Systems Approach to Development: Applications. Cambridge, MA: Bradford Books/MIT Press Sternberg, R. (1997). Inteligencia exitosa. Como una inteligencia practica y creativa determina el éxito en la vida. Barcelona. Paidós.

Stobart, G. (2010). Tiempos de pruebas. Los usos y abusos de la evaluación. Madrid. Morata

Tomasello, M. (2010). ¿Por qué cooperamos? Madrid: Katz Editores.

Tomasello, Michael. (2013). Los orígenes de la comunicación humana. Madrid: Katz Editores.

Vygotsky, L. (1979). El desarrollo de los procesos psicológicos superiores. Barcelona: Editorial Crítica.

Zamagni, S. (2009). Economía del bien común. Madrid: Editorial Ciudad Nueva.

Wertheimer, M. (1991). El pensamiento productivo. Barcelona. Editorial Paidós Ibérica. 